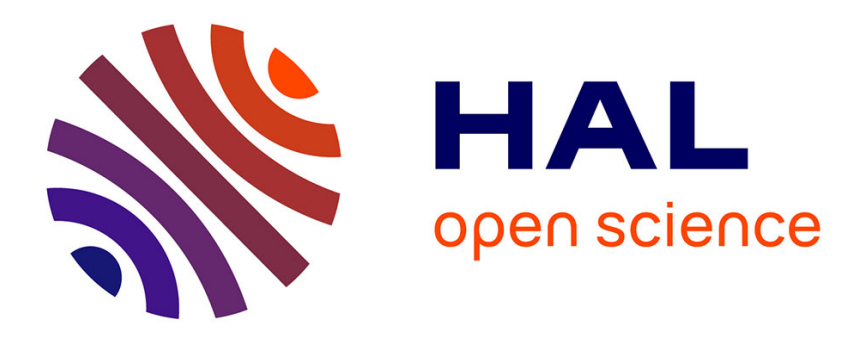

\title{
A new algorithm based CSP framework for RFID network planning
}

\author{
Atef Jaballah, Aref Meddeb
}

\section{To cite this version:}

Atef Jaballah, Aref Meddeb. A new algorithm based CSP framework for RFID network planning. Journal of Ambient Intelligence and Humanized Computing, 2020, 1, 10.1007/s12652-020-02446-5 . hal-02985156

\section{HAL Id: hal-02985156 https://hal.science/hal-02985156}

Submitted on 3 Nov 2020

HAL is a multi-disciplinary open access archive for the deposit and dissemination of scientific research documents, whether they are published or not. The documents may come from teaching and research institutions in France or abroad, or from public or private research centers.
L'archive ouverte pluridisciplinaire HAL, est destinée au dépôt et à la diffusion de documents scientifiques de niveau recherche, publiés ou non, émanant des établissements d'enseignement et de recherche français ou étrangers, des laboratoires publics ou privés. 


\title{
A new algorithm based CSP framework for RFID network planning
}

\author{
Atef Jaballah • Aref Meddeb
}

\begin{abstract}
The huge growth of industrial society requires the deployment of radio frequency identification networks on a large scale. This necessitates the installation of a large number of radio frequency identification components (readers, tags, middleware and others). As a consequence, the cost and complexity of networks are increasing due to the large number of readers to be installed. Finding the optimal number, placement and parameters of readers to provide a high quality of service for radio frequency identification systems is a critical problem. A good planning affords a basic need for radio frequency identification networks, such as coverage, load balance and interference between readers. This problem is famous in the literature as a radio frequency identification network planning problem. All the proposed approaches in the literature have been based on meta-heuristics. In this paper, we design a new algorithm, called the RNP-CSP algorithm based on the constraint satisfaction problem framework to solve the radio frequency identification network planning problem. The performance evaluation shows that the RNP-CSP algorithm is more efficient than $P S^{2} O$, GPSO and VNPSO-RNP.
\end{abstract}

Keywords radio frequency identification · network planning · constraint satisfaction problem

\section{Introduction}

Due to the multiple advantages of Radio Frequency IDentification (RFID) technology (no physical contact, rapidity, high security and high storage capacity), and unlike the traditional barcode identification technology, RFID is widely used to tag

Atef Jaballah

LORIA, UMR 7503, Lorraine University, Nancy, France

E-mail: atef.jaballah@loria.fr

Aref Meddeb

National Engineering School of Sousse, University of Sousse. Tunisia NOCCS Laboratory : Networked Objects Control \& Communication Systems

E-mail: Aref.Meddeb@infcom.rnu.tn 
and identify physical objects in different fields such as: industry, healthcare, supply chain management and transportation Zhu et al. (2012); Jaballah and Meddeb (2017a); Aboelmaged and Hashem (2018); Ruan et al. (2018); Talib et al. (2020). RFID is often viewed as a key technology of the IoT Meddeb (2016). Indeed, RFID allows providing location-based information for large-scale IoT applications Figueiredo e Silva et al. (2018); Elbasani et al. (2020). An RFID network consists of a set of tags, one or several RFID readers, and a computer system linked to readers. Usually in an RFID system, the tags are distributed in a large-scale working area such as a warehouse or an airport. Owing to the limited communication range of the RFID antennas, multiple readers should be deployed in the working area to cover all the tags. Then, we need to determine the optimal number of readers, their coordinates and their parameters to assure full tag coverage. This problem is known in the literature as the RFID Network Planning (RNP) problem. RNP aims to optimize multiple objectives, subject to specific constraints such as coverage, load balance and interference between readers. Solving the RNP problem is an NP-hard task. In fact, RNP is large-scale, high-dimensional, nonlinear optimization problems with large number of variables and uncertain parameters. Due to the complexity of the RNP problem, it cannot be tackled by the traditional optimization methods.

In the literature, there have been several approaches based on the metaheuristics model to solve the RNP problem. However, meta-heuristics are often able to find the right solutions, but we cannot guarantee that the found solutions are optimal. In this work, we propose a new RNP-CSP (Constraint Satisfaction Problem) algorithm to solve this problem. The advantages of our work are summarized as follows: $(i)$ We formulate the RNP optimization problem as a CSP. On the other hand, we transform the multi-objectives optimization problem (NP-hard) to a decision problem (NP-complete) i.e. a problem which may be answered by "yes" or "no". The decision version of the RNP problem is easier than (or in the worst case, the same as) the optimized version. (ii) The modeling of the RNP problem as a discrete CSP reduces the search space, which speeds up the search process and avoids placing readers in front of obstacles in order to shun interference. (iii) Our approach is able to adjust the number of RFID readers during the research process, unlike the majority of the methods described in the literature.

The rest of this paper is organized as follows. Section 2 presents related work. Section 3 forms the RNP problem. Section 4 describes our proposed algorithm based on the CSP framework for solving the RNP problem. We evaluate the proposed algorithm in section 5 . We conclude our discussion in section 6 .

\section{Related work}

Meta-heuristics represent a natural framework to solve complex problems, particularly the RNP problem. The Genetic Algorithm (GA) has been commonly used to address the RNP problem. In Guan et al. (2006), the authors suggested a genetic approach for RNP, where a genome represented all information of the RFID network. The genome was encoded hierarchically: The first level decided the choice of candidate sites. The second level determined the type of antennas. The last level handled the parameters of the reader placement. The crossover operator was applied to the first level of the encoding hierarchy, and the mutation operator 
was at all levels of the hierarchy. In Yang et al. (2009), the authors used binary coding to represent the information of readers (location and coverage) and real coding to express the physical attributes of antennas (type, power, azimuth). For individual selection, the combination of roulette and championship was used. The cross and mutation operators were also utilized to maintain population diversity. The writers in Zhang et al. (2019) introduced the directional antenna reader with the omnidirectional antenna reader to improve RNP using the GA.

H. Chen et al. Hanning et al. (2011) put forward the multi-swarm particle swarm optimizer $P S^{2} O$ algorithm to optimize RNP. The main idea of $P S^{2} O$ was to extend the single population PSO to the interacting multi-swarm model by constructing a hierarchical interaction topology and enhancing dynamical update equations. $P S^{2} O$ was utilized to optimize the tag coverage, the interference level, the economic efficiency and the load balance. In Chen and Zhu (2008) the Evolutionary Strategy (ES) and the PSO are applied to the RNP problem. A PSO algorithm with the Von Neumann topology (VNPSO-RNP) was proposed in Gong et al. (2012) to optimize RNP. VNPSO-RNP adjusted the number of deployed readers pending the optimization process thanks to the tentative reader elimination operator. In Dimitriou et al. (2019), the authors introduced hybrid PSO which included an additional velocity vector to optimize the locations and the configurations of RFID readers. PSO was also combined with the Estimation of Distribution Algorithm (EDA) in Gao et al. (2010) to solve multi-objective RNP, where individuals were divided into two partitions: The first was managed by the EDA that used global information to explore the search space. The second partition was controlled by the PSO algorithm, which utilized local information to exploit the search space. The authors used a balance parameter to maintain the equilibrium between exploration and exploitation. In Dimitriou et al. (2019) and Gao et al. (2010), the coverage, the interference level and the economic efficiency were taken into account when deploying the RFID network. In Zhu and Li (2018), the GA and the PSO are directly applied to deploy RFID readers for the surveillance of predictable mobile objects. A Robust PSO (RPSO) was developed in Xu et al. (2018) to plan a network in logistics under uncertainties where the probability sensing model was utilized to determine the coverage rate, and the Monte Carlo approach was used to analyze the interference between readers.

Jaballah et al. Jaballah and Meddeb (2017b); Jaballah and Meddeb (2017) put forward a new variant of Cuckoo Search (CS) called Self-Adaptive CS (SACS) algorithm; to solve the RNP problem. The SACS algorithm adjusted the control parameters of the original CS algorithm dynamically in real-time, to strike a balance between exploration and exploitation during the different evolving phases. Five objectives which were: the coverage, the number of deployed readers, the interference level, the load balance and the power loss, were considered during the optimization process. Gradient-Based CS was proposed in Hasnan et al. (2019); hasnan et al. (2019) to optimize several objectives in RNP problems such as: the number of readers, the interference and the tag coverage.

The Bacteria Foraging (BFO) algorithm has been widely used to resolve the RNP problem. A variant of BFO, called Multi-Colony BFO (MC-BFO), was proposed in Chen et al. (2010) to solve the RNP problem. This variant was not based on the chemotaxis behavior of canonical BFO only, but it is also based on the bacterial communication mechanisms of the multi-colony bacterial community. MC-BFO combined the coverage, the interference level,the economic ef- 
ficiency and the load balance to configure the RFID network. In Yuan et al. (2018) a novel Indicator-based Multi-Objective Bacterial Colony foraging Algorithm (I-MOBCA) was put forward to tackle the RNP problem. The I-MOBCA combined various strategies: bacterial foraging, cell-to-cell communication, preference indicator-based measure, and adaptive searching, to optimize three objective of the RNP problem.

In addition, numerous papers have extended the Artificial Bee Colony (ABC) algorithm to solve the RNP problem. In Ma et al. (2019), A multiobjective evolutionary algorithm based on the ABC, namely the H-MOABC, was developed to find optimal planning of RFID networks. A hybridization between the ABC and heuristics was applied in Nebojsa Bacanin and Strumberger (2015) to find the optimal number and locations of RFID readers. In Ma et al. (2014), a Hierarchical ABC (HABC) was designed by extending the single ABC algorithm to the hierarchical and cooperative mode by merging the comprehensive learning method and the multi-population cooperative co-evolution approach. The HABC was utilized to resolve the real-world RNP problem. A hybrid algorithm, called (GI-ABC), combining the GA and the ABC was put forward in Tuba et al. (2015) for this problem. In Tuba and Bacanin (2015), the canonical Bat algorithm (BA) was hybridized with the ABC for tackling the large-scale RNP problem. The performance of the BA was improved by integrating an onlooker bee mechanism, and the resulting algorithm was termed the (BA-OM).

In Zhang and Liu (2017), a Curling Algorithm for RNP (CA-RNP) was designed to solve the RNP problem. CA-RNP would implement the reader-movement and reader-collision operators to determine the optimal number of deployed readers and the best position of readers in the monitoring area. Coverage, interference load balance and power were also considered by CA-RNP. A decomposition-based firefly algorithm was suggested in Zhao et al. (2017) for a multi-objective RNP optimization problem. The authors combined the virtual force and the random walk to enhance exploitation and optimize these objectives: the number of deployed readers, coverage and the interference level. Self Learned Invasive Weed-Mixed Biogeography Based Optimization (SLIWMBBO) was proposed in Zahran et al. (2020) to solve large scale and highly dense RNP instances .

Localized reader scheduling algorithms were suggested in Campioni et al. (2019) to maximize the networking lifetime and the tag coverage for RFID networks. Zhang et al Zhang et al. (2018) developed an integer linear programming to identify the optimal placement of base stations and RFID-reader-embedded roadside units at roadsides in VANETs to enhance road safety. In Shi et al. (2018), an improved of Chicken Swarm Optimization (CSO) algorithm, named MPCSO, was proposed to deploy the directional reader antennas in RFID networks.

In this paper, we show that the RNP problem can be placed within the framework of CSPs, unlike the state-of-the-art solutions based on meta-heuristics.

\section{Network planning in RFID systems}

In this section, we give some necessary background for understanding the RNP problem. 
3.1 Working area and search space

An RFID network is generally defined on a rectangular workspace denoted $\mathcal{A}$, as shown in Fig. 1. The first step for solving the RNP problem is to determine the search space. The latter represents all positions possible to place RFID readers. Two search space models are available:

- Continuous search space: In this case, an infinite number of RFID-reader positions are possible. In other words, RFID readers can be placed anywhere in the working area. The decision variables for the RNP problem are the position coordinates of RFID readers in a bi-dimensional space. When planning the RFID network, the readers will be placed next to each other to find the optimal configuration among an infinite configuration possible.

- Discrete search space: In this case, the RFID-reader positions are represented by a finite set of candidate sites as determined before the optimization phase. Contrary to the continuous search space, the number of possible configurations is countable. Let $n$ be the number of candidate sites and $k$ be the number of RFID readers to deploy in the working area. Thus, we have $\left(\begin{array}{l}n \\ k\end{array}\right)=\frac{n !}{(n-k) ! k !}$ possible configurations. For example, to select the placement of 100 readers from 1, 000 candidate sites, there are approximately $100^{300}$ configurations possible, where $k \ll n \sim O\left(n^{k}\right)$.

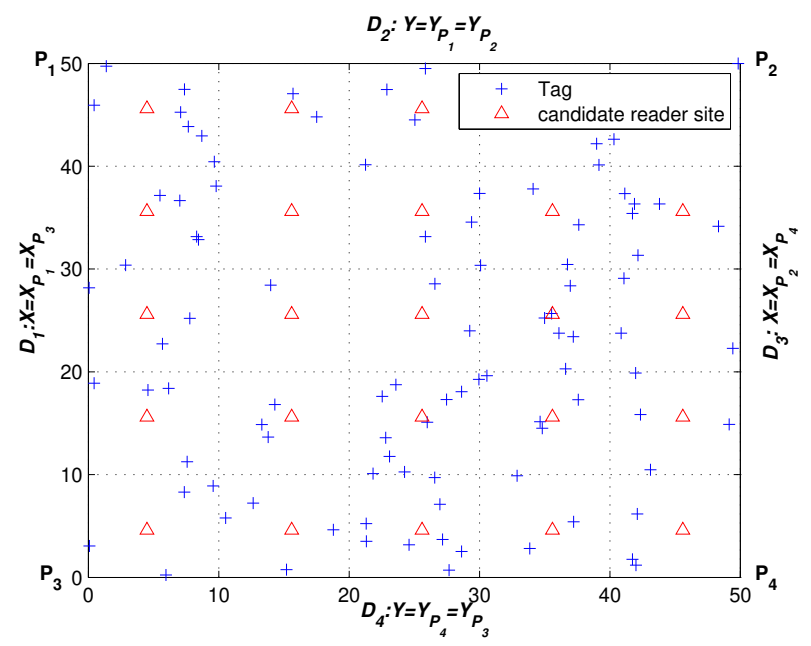

Fig. 1 Working area of approximately $50 \mathrm{~m}$ by $50 \mathrm{~m}$ with 100 RFID tags distributed uniformly, and 25 reader candidate sites.

In this work, we have chosen a discrete search space to solve the RNP problem. The following reasons justify our choice: firstly, the discrete model reduces the size of the search space. Consequently, it accelerates the optimization process. Moreover, radio wave propagation can be calculated as a priory, which reduces the computing time. In addition, this model requires a preprocessing phase to define 
the set of candidate sites. During this phase, we use the architectural characteristics of the working area to avoid placing the readers in front of obstacles that cause an attenuation of the transmitted waves, hence avoiding the RFID network quality of service degradation.

\subsection{Communication in RFID network}

An RFID network consists of three components: a tag or transponder on which an IDentifier (ID) is stored, a reader that collects the IDs from tags, and a middleware that interprets the information provided by readers. This information can be stored on servers for analysis and sharing. RFID tags can be classified into three types according to their power source: passive, semi-passive and active. Passive tags do not have any internal power source, and they are powered periodically through the electromagnetic energy transmitted by an RFID reader. Passive tags are characterized by their low cost and by their limited range, which does not go beyond 10 meters. Semi-passive tags utilize a battery to run the embedded chip only. The communication is done thanks the electromagnetic energy of a reader. Semi-passive tags are more expensive than passive tags. Active tags have their own power source, which is usually a battery. The latter is utilized to run the embedded circuit and to broadcast the signal to readers. Active tags afford a much longer read range than passive tags, which can reach 100 meters, but they are also more costly than passive tags. Due to the lower cost of passive tags, passive RFID systems are more deployed than active RFID systems.

Table 1 Notation of RNP formulation

\begin{tabular}{ll}
\hline Symbol & Representation \\
\hline \hline SCS & Set of candidates sites on which readers may be placed \\
\hline $\mathrm{TS}$ & Set of tags distributed in $\mathcal{A}$ \\
\hline $\mathrm{RS}$ & Set of readers deployed in $\mathcal{A}$ \\
\hline $\mathrm{W}$ & Number of candidates sites $=|S C S|$ \\
\hline $\mathrm{M}$ & Number of tags $=|T S|$ \\
\hline $\mathrm{N}$ & Number of readers $=|R S|$ \\
\hline$T h_{T}$ & Tag sensitivity \\
\hline$T h_{R}$ & Reader sensitivity \\
\hline$r_{i}$ & Interference range of reader $R_{i}$ \\
\hline$d_{R_{i}, R_{j}}$ & Euclidean distance between two adjacent readers $R_{i}$ and $R_{j}$. \\
\hline$\beta$ & Relaxation coefficient of load balancing constraint \\
\hline$\gamma$ & Tolerated interference rate \\
\hline$C_{m a x}$ & Reader capacity (maximum number of tags which can be read by the reader) \\
\hline
\end{tabular}

For this reason, this work is limited to the study of passive RFID systems that use backscatter communication. The notations used in this paper are listed in Table 1. In backscatter RFID systems, a bidirectional radio link is established between the reader and the tag to guarantee the half-duplex communication. A bidirectional radio is composed of two links: forward and backward. In the forward link, the reader transmits the $\mathrm{RF}$ power, $P_{\text {trans }(R)}$, and data to the tag. The tag chip absorbs this power. If the latter is greater than the chip sensitivity $T h_{T}$, the chip is activated and the tag is ready to reply. Else, communication is limited to 
the forward link. The power accessible to the tag is determined according to the Friis transmission equation:

$$
P_{\operatorname{recv}(T)}[d B m]=P_{\operatorname{trans}(R)}[d B m]+G_{R}[d B i]+G_{T}[d B i]-L F[d B]
$$

where $P_{\text {trans }(R)}$ is the power radiated by the reader antenna, $G_{R}$ is the reader antenna gain, $G_{T}$ is the tag antenna gain, and $L F$ is the free-space path loss, given by:

$$
L F[d B]=10 \log \left[\left(\frac{4 \Pi}{\lambda}\right)^{2} d^{n}\right]+\delta[d B]
$$

Usually, an RFID system is installed in indoor environments, so the multipath loss is treated. In equation (2), $\lambda$ is the wavelength, $d$ is the Euclidean distance between the two devices, $n \in[1.5,4]$ due to medium conditions, and $\delta$ represents other losses due to the pluralization loss and the cable loss. In the backward link, the tag responds to the reader by modulating the backscattered signal. The backscattered power $P_{b}$ is written as $P_{b}=\Gamma_{t a g}{ }^{2} P_{\operatorname{recv}(T)}$, where $\Gamma_{t a g}$ is the tag reflection coefficient and $P_{\operatorname{recv}(T)}$ is the power received by the tag. The power received by the reader, $P_{\operatorname{recv}(R)}$, should be higher than its sensitivity $T h_{R}$ to ensure the backward link. $T h_{R}$ is defined as the minimum energy necessary to guarantee the reception of data by the reader. Formally, $P_{\operatorname{recv}(R)}$ is defined by:

$$
P_{r e c v(R)}[d B m]=P_{b}[d B m]+G_{R}[d B i]+G_{T}[d B i]-L B[d B]
$$

with

$$
L B[d B]=20 \log \left(\frac{4 \Pi d}{\lambda}\right)
$$

\section{Constraint satisfaction problem formulation for RFID network planning}

Contrary to the related work based on meta-heuristics, we propose in this paper a new algorithm, called the RNP-CSP algorithm, based on the CSP framework, to solve the RNP problem. The main idea of our algorithm is to transform the multiobjective optimization RNP problem into a CSP. The latter is defined by a set of variables, a set of domains and a set of constraints Barto et al. (2017). Each variable has a nonempty domain of possible values. Each constraint involves a subset of variables and specifies the allowable combinations of values for that subset. This subset of variables is called the scope of this constraint. The arity of a constraint is the size of its scope. The arity of a problem is the maximum arity of its constraints. Instantiation is the assignment of a value to each variable, and it is said to be complete if it fixes all variables. The instantiation that is not complete is said to be partial. Partial instantiation is consistent if it satisfies all of the constraints whose scopes have no uninstantiated variables. A CSP solution is complete, consistent instantiation.

The RNP problem can be modeled as a CSP. We define a set of variables $V=\left\{\left\langle X_{R_{j}}, Y_{R_{j}}\right\rangle, P_{R_{j}}, T_{i, j}\right\}$, where $\left\langle X_{R_{j}}, Y_{R_{j}}\right\rangle$ represents the coordinates of the RFID reader $R_{j}$ in the workspace $\mathcal{A}, P_{R_{j}}$ is the power level transmitted by $R_{j}$, 
and $T_{i, j}$ is a binary variable that indicates whether $\operatorname{tag} T_{i}$ is covered by reader $R_{j}$ or not.

We associate a finite domain for each variable, and we note by $D$ the domain of these variables $D=\left\{D_{\left\langle X_{R_{j}}, Y_{R_{j}}\right\rangle}, D_{P_{R_{j}}}, D_{T_{i, j}}\right\}$, where $D_{\left(X_{R_{j}}, Y_{R_{j}}\right)}=S C S$ represents the domain of the $\left\langle X_{R_{j}}, Y_{R_{j}}\right\rangle$ variable, $\forall R_{j} \in R S, D_{P_{R_{j}}}$ corresponds to the domain of the $P_{R_{j}}$ variable, $\forall R_{j} \in S R$, and $D_{T_{i, j}}=\{0,1\}$ represents the domain of the $T_{i, j}$ variable, $\forall T_{i} \in T S, \forall R_{j} \in R S$.

We also define a set of constraints $C=\left\{C_{1}, C_{2}, C_{3}, C_{4}, C_{5}, C_{6}\right\}$ to meet the RNP requirements (See Fig. 2), where $C_{1}$ ensures that each tag is covered by a reader at least, $C_{2}$ ensures that the power received by the tag is higher than its sensitivity, thus guaranteeing the forward link, $C_{3}$ ensures that the power received by the reader is higher than its sensitivity in order to guarantee the backward link, $C_{4}$ ensures the load balance between different readers deployed in $\mathcal{A}, C_{5}$ guarantees that the network interference does not exceed the tolerated rates of interference, and $C_{6}$ prevents two readers or more from being placed on the same site. $C_{1}, C_{2}$ and $C_{3}$ check the link budget model.

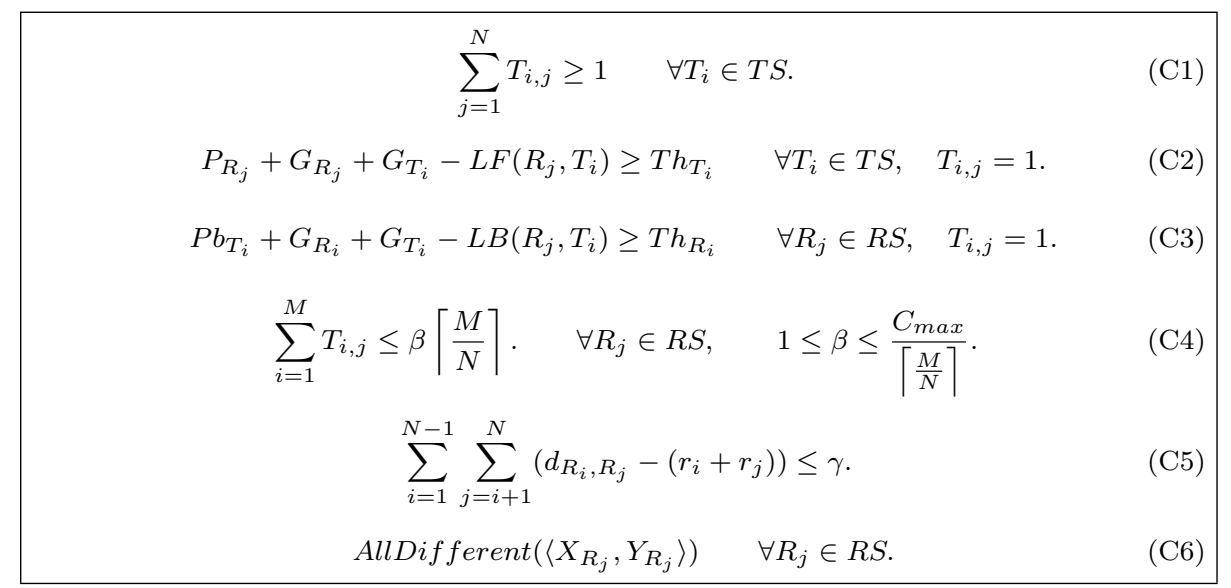

Fig. 2 RNP-CSP model

The RNP problem is represented by an $M$-ary CSP, as represented in Fig, 2 , Knowing that $N \ll M$, the RNP-CSP can be represented as a CSP instance with $2 N+M \times N$ variables and $2(N \times M)+N+M+2$ constraints as shown in Table 2.

Our algorithm described by flowchart in Fig. 3 takes as an input two sets of parameters: The first set of inputs includes the set of candidate sites on which readers may be placed, the tag localization and the structure of the working area. The second set of inputs consists of the linked budget, the relaxation coefficient of the load balancing constraint $\beta$, and the tolerated interference rate $\gamma$. The network designer fixes these latter. This task allows the network designer to specify the cost of network deployment, depending on the type and scope of the RFID system. Our algorithm is made of three main parts. The first part involves determining 
Table 2 Characteristic of RNP-CSP model

\begin{tabular}{lll}
\hline Constraint & Arity & Number \\
\hline \hline C1 & $\mathrm{N}$ & $\mathrm{M}$ \\
\hline $\mathrm{C} 2$ & 2 & $M \times N$ in worst cases \\
\hline $\mathrm{C} 3$ & 2 & $N \times M$ in worst cases \\
\hline $\mathrm{C} 4$ & $\mathrm{M}$ & $\mathrm{N}$ \\
\hline $\mathrm{C} 5$ & $\mathrm{~N}(\mathrm{~N}-1)$ & 1 \\
\hline $\mathrm{C} 6$ & $\mathrm{~N}$ & 1 \\
\hline \hline & Maximum arity $=\mathrm{M}$ & $e=2(N \times M)+N+M+2$ \\
\cline { 2 - 3 }
\end{tabular}

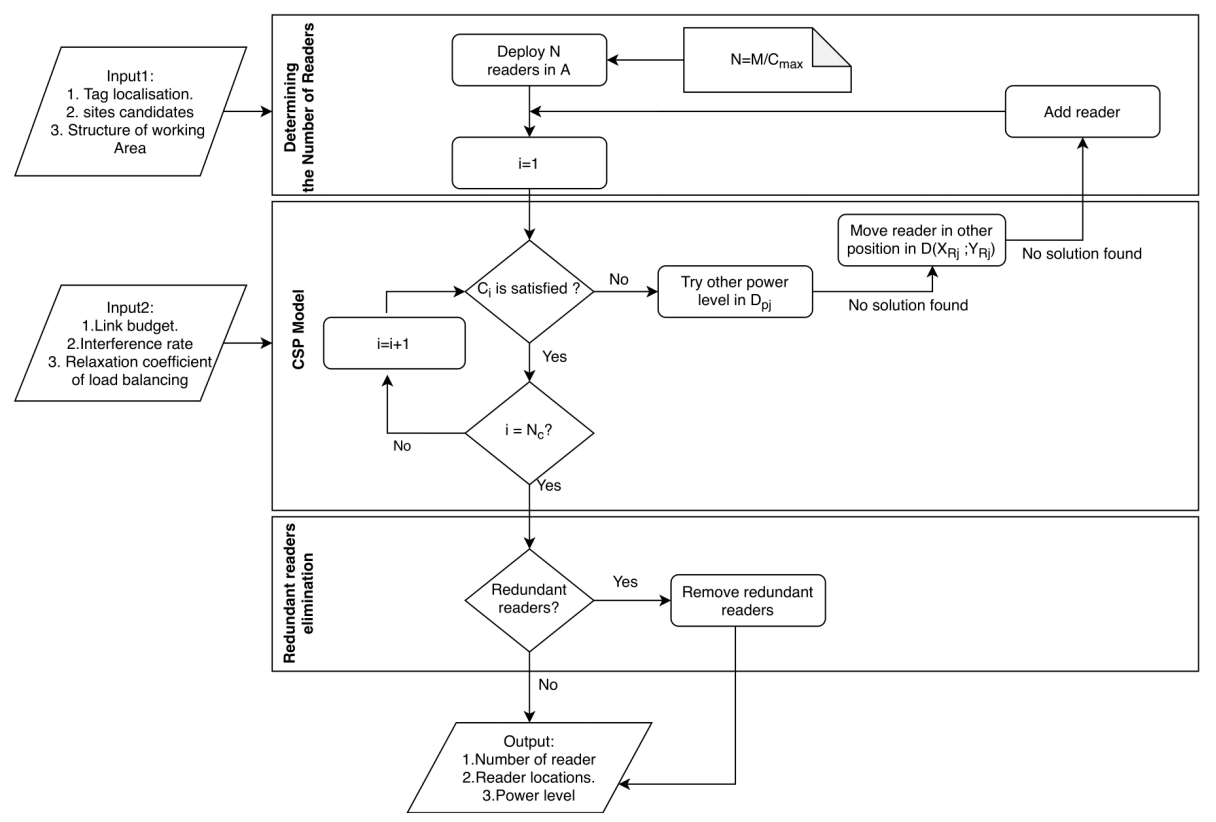

Fig. 3 Flow chart of RNP-CSP algorithm

the minimum number of readers necessary to cover all tags distributed in the working area. The second part represents the solution algorithm to solve the RNPCSP model. In this model, each of these objectives: coverage, load balance and interference between readers is transformed into one or several constraints. Once all constraints are satisfied, the third party guarantees the elimination of redundant readers that increase the interference level and the cost of network deployment.

\section{Experimental results}

For our experiments, tags are distributed in a $50 \mathrm{~m} \times 50 \mathrm{~m}$ working area. Six benchmarks instances: C30, C50, C100, R30, R50, and R100 are tested. By default, the number of candidate sites is 500. In the first three instances, the tags are 
clustered. For other instances, the tags are distributed uniformly. Reader, tag and linked budget specification used in this simulation are presented in Table 3.

Table 3 Reader, tag and link budget specification

\begin{tabular}{llllll} 
Reader specification & & Tag specification & \multicolumn{2}{l}{ Link Budget specification } \\
\hline \hline Radiated power & $20-33 \mathrm{dbm}(0.1-2 \mathrm{w})$ & Tag reflection coefficient & 0.3 & Wavelength & $0.328 \mathrm{~m}$ \\
Reader sensitivity & $-80 \mathrm{dBm}$ & Tag sensitivity & $-14 \mathrm{dBm}$ & Medium conditions & 2 \\
Gain of antenna & $6.7 \mathrm{dBi}$ & Gain of antenna & $3.7 \mathrm{dBi}$ & Wireless communication losses & 2 \\
\hline
\end{tabular}

In order to evaluate the performance of our RNP-CSP approach, we have implement our algorithm using the constraint programming tool CHOCO Prud'homme et al. (2017) and compare it with the best results found by canonical PSO and two variants of the PSO algorithm, namely $P S^{2} O$ and VNPSO-RNP, developed specifically to solve the RNP problem, after 50 independent runs. For canonical PSO and these variants, we set the population size to 20 and the maximum number of generations to 10,000 . For $P S^{2} O$, the population is divided into four swarms of the same size 5 . The remaining configuration has been the same as Hanning et al. (2011). For PSO and VNPSO-RNP, we use the same configuration described in Gong et al. (2012). For the CSP-RNP algorithm, $\gamma$ is fixed to zero, $\beta$ is set at 1.7 and the maximum reader capacity is fixed to 50 tags. The choice of these values promotes the search for solutions that deploy the minimum number of readers without interference, which are the most important objectives in the RNP problem Ma et al. (2019), due to the high costs of RFID readers and the impact of the interference on the quality of service in the RFID system.

Table 4 shows the experimental results for clustered instances. For instance C_30, all algorithms can guarantee full tag coverage without interference. Furthermore, the CSP-RNP algorithm utilizes fewer RFID readers than the other tested algorithms, as illustrated in Fig. 4. The CSP-RNP algorithm is also more efficient in terms of load balancing than the other methods, for instance $C_{-} 30$. Indeed, Fig. 6 depicts that the standard deviation between the number of tags monitored by each reader in the CSP-RNP algorithm is much smaller than that in other algorithms. For instances, C_50 and C_100, all tested algorithms can achieve full tag coverage, but our algorithm requires fewer readers to accomplish this goal, which reduces the cost and complexity of these networks.

Table 4 Results of four algorithms for solving clustered instances: C_30,C_50 and C_100

\begin{tabular}{|c|l|l|l|l|l|}
\hline Benchmarks & Algorithms & Coverage & Readers & Interferences & Load balancing \\
\hline \hline \multirow{3}{*}{ C_30 } & PSO & $100.00 \%$ & 6 & 0 & $1.0582 \mathrm{e}-4$ \\
\cline { 2 - 6 } & $P S^{2} O$ & $100.00 \%$ & 4 & 0 & $5.6689 \mathrm{e}-4$ \\
\cline { 2 - 6 } & VNPSO-RNP & $100.00 \%$ & 3 & 0 & $1.1904 \mathrm{e}-3$ \\
\cline { 2 - 6 } & RNP-CSP & $100.00 \%$ & 2 & 0 & $4.4642 \mathrm{e}-3$ \\
\hline \hline \multirow{3}{*}{ C_50 } & PSO & $100.00 \%$ & 6 & 0 & $1.2210 \mathrm{e}-5$ \\
\cline { 2 - 6 } & $P S^{2} O$ & $100.00 \%$ & 6 & 0 & $3.9246 \mathrm{e}-6$ \\
\cline { 2 - 6 } & VNPSO-RNP & $100.00 \%$ & 5 & 0 & $1.4652 \mathrm{e}-5$ \\
\cline { 2 - 6 } & RNP-CSP & $100.00 \%$ & 4 & 0 & $7.4321 \mathrm{e}-5$ \\
\hline \hline \multirow{3}{*}{ C_100 } & PSO & $100.00 \%$ & 6 & 0 & $3.3099 \mathrm{e}-8$ \\
\cline { 2 - 6 } & $P S^{2} O$ & $100.00 \%$ & 5 & 0 & $5.5087 \mathrm{e}-7$ \\
\cline { 2 - 6 } & VNPSO-RNP & $100.00 \%$ & 5 & 0 & $2.9239 \mathrm{e}-6$ \\
\cline { 2 - 6 } & RNP-CSP & $100.00 \%$ & 4 & 0 & \\
\hline
\end{tabular}




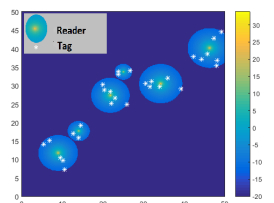

(a)

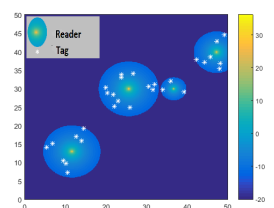

(b)

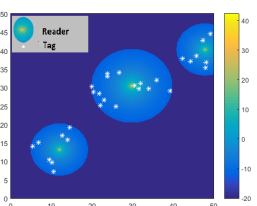

(c)

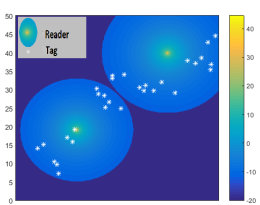

(d)

Fig. 4 Comparison of reader location and received power distribution for C30 obtained by: (a) PSO, (b) $P S^{2} O$, (c) VNPSO-RNP, (d) CSP-RNP

As it can be seen in Table 5 only the CSP-RNP algorithm succeeds in ensuring full tag coverage without interference between readers in all uniformed instances. For R_30, our algorithm uses two readers to provide full tag coverage without interference, but the other algorithms use six readers to achieve the same goal. When solving R_50, PSO cannot reach full tag coverage in its best runs among 50 . On the contrary, $P S^{2} O$ and VNPSO-RNP can obtain $100 \%$ coverage, but with a high number of readers and a high level of interference. Exclusively, the CSP-RNP algorithm can provide full tag coverage, without interference and a small number of readers. All these solutions are detailed in Fig. 5. The load balancing between the different readers for the previous solutions is illustrated in Fig.6. It is clear that the CSP-RNP outperforms the other algorithms in terms of load balancing since the numbers of tags monitored by each reader in the CSP-RNP solution are the closest. For instance R_100, only CSP-RNP and VNPSO-RNP are able to provide full tag coverage. Nevertheless, CSP-RNP is significantly superior to VNPSO-RNP in terms of number of deployed readers, interference and load balance. According to these experimental results, we conclude that the CSP-RNP algorithm is greatly superior to its counterparts for all instances in terms of all comparative metrics. Consequently, the RNP-CSP algorithm can significantly improve the high-quality of service in RFID systems. Although our algorithm outperforms the state-of-theart solution in terms of quality of solution, it is slower in terms of convergence speed.

\section{Conclusion}

In this paper, we put forward a new RNP approach called the CSP-RNP framework. The multiobjective optimization RNP problem is transformed to a CSP. The resulting CSP is solved using a brute force search. The simulation results show that the RNP-CSP outperforms the state-of-the-art solutions. Moreover, the CSP-RNP framework is expandable and we can easily add other RNP objectives.

In this paper, we have limited our work to study the optimization in a passive RFID system. In the future, we will extend our approaches to semi-passive and active RFID systems, where several other constraints and objectives will be imposed due to the energy source of tags. Moreover, for RFID systems, there are often obstacles between readers and tags that cause reflections of the RF signal sent by the reader. This reflection results in a variation in the form of the reader interrogation zone, thus the interference. This problem should be considered during RNP in the future work. Furthermore, in industry 4.0, mobile RFID networks 
are progressively deployed. Readers move to track products in the manufacturing line. The movement of readers causes interference when a reader passes alongside another reader. To overcome this problem, the speed and the motion sector of each reader must be determined. Dynamic RNP is another potential direction for our work.

Table 5 Results of four algorithms for solving uniformed instances: R_30,R_50 and R_100

\begin{tabular}{|l|l|l|l|l|l|}
\hline Benchmarks & Algorithms & Coverage & Readers & Interferences & Load balancing \\
\hline \hline \multirow{3}{*}{ R_30 } & PSO & $100.00 \%$ & 6 & 0 & $8.5034 \mathrm{e}-5$ \\
\cline { 2 - 6 } & $P S^{2} O$ & $100.00 \%$ & 6 & 0.0480 & $8.3333 \mathrm{e}-5$ \\
\cline { 2 - 6 } & VNPSO-RNP & $100.00 \%$ & 6 & 0.0223 & $7.9365 \mathrm{e}-5$ \\
\cline { 2 - 6 } & RNP-CSP & $100.00 \%$ & 2 & 0 & $3.4722 \mathrm{e}-4$ \\
\hline \hline \multirow{3}{*}{ R_50 } & PSO & $98.00 \%$ & 6 & 0.0864 & $4.6850 \mathrm{e}-6$ \\
\cline { 2 - 6 } & $P S^{2} O$ & $100.00 \%$ & 8 & 0.1905 & $1.0433 \mathrm{e}-6$ \\
\cline { 2 - 6 } & VNPSO-RNP & $100.00 \%$ & 7 & 0.1493 & $1.7669 \mathrm{e}-6$ \\
\cline { 2 - 6 } & RNP-CSP & $100.00 \%$ & 5 & 0 & $1.3997 \mathrm{e}-5$ \\
\hline \hline \multirow{3}{*}{ R_100 } & PSO & $95.00 \%$ & 6 & 0.1232 & $8.8605 \mathrm{e}-8$ \\
\cline { 2 - 6 } & $P S^{2} O$ & $97.00 \%$ & 6 & 0.3082 & $8.8284 \mathrm{e}-8$ \\
\cline { 2 - 6 } & VNPSO-RNP & $100.00 \%$ & 8 & 0.2708 & $1.2978 \mathrm{e}-8$ \\
\cline { 2 - 6 } & RNP-CSP & $100.00 \%$ & 6 & 0 & $108548 \mathrm{e}-7$ \\
\hline \hline
\end{tabular}

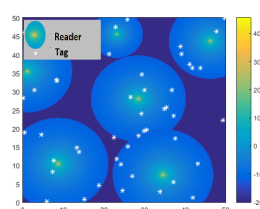

(a)

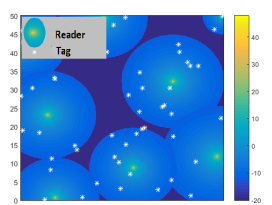

(b)

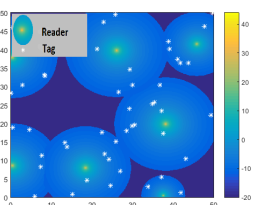

(c)

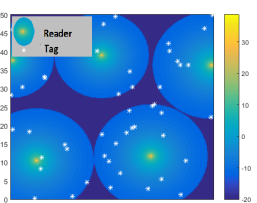

(d)

Fig. 5 Comparisons of reader location and received power distribution for R50 obtained by: (a) PSO, (b) $P S^{2} O$, (c) VNPSO-RNP, (d) CSP-RNP

\section{References}

Aboelmaged M, Hashem G (2018) RFID application in patient and medical asset operations management: A technology, organizational and environmental (TOE) perspective into key enablers and impediments. International Journal of Medical Informatics 118:58 - 64

Barto L, Krokhin A, Willard R (2017) Polymorphisms, and How to Use Them. In: Krokhin A, Zivny S (eds) The Constraint Satisfaction Problem: Complexity and Approximability, Dagstuhl Follow-Ups, vol 7, Schloss Dagstuhl-LeibnizZentrum fuer Informatik, pp 1-44

Campioni F, Choudhury S, Al-Turjman F (2019) Scheduling RFID networks in the IoT and smart health era. Journal of Ambient Intelligence and Humanized Computing 10(10):4043-4057, doi:10.1007/s12652-019-01221-5, URL https:// doi.org/10.1007/s12652-019-01221-5 


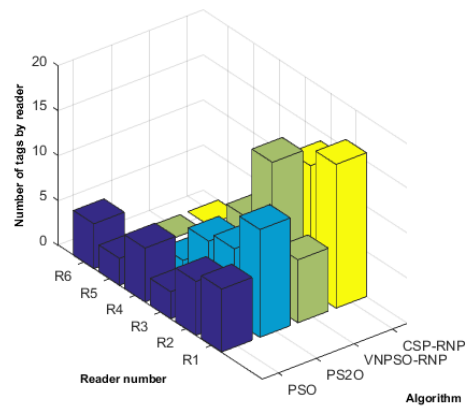

(a)

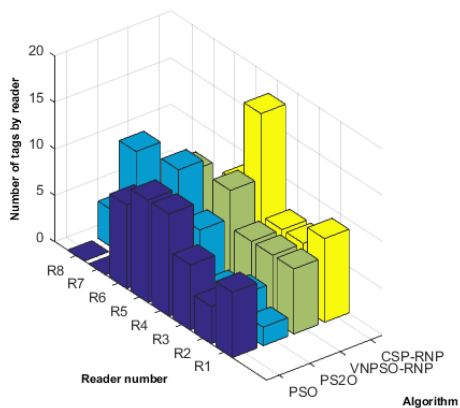

(b)

Fig. 6 Load balancing obtained by all algorithms for instances (a) C_30 (b) R_50

Chen H, Zhu Y (2008) Rfid networks planning using evolutionary algorithms and swarm intelligence. In: 2008 4th International Conference on Wireless Communications, Networking and Mobile Computing, pp 1-4

Chen H, Zhu Y, Hu K (2010) Multi-colony bacteria foraging optimization with cell-to-cell communication for RFID network planning. Appl Soft Comput 10(2):539-547

Dimitriou AG, Siachalou S, Bletsas A, Sahalos J (2019) Introduction of dynamic virtual force vector in particle swarm optimization for automated deployment of RFID networks. In: 2019 13th European Conference on Antennas and Propagation (EuCAP), pp 1-5

Elbasani E, Siriporn P, Choi JS (2020) A Survey on RFID in Industry 4.0. Springer International Publishing, doi:10.1007/978-3-030-32530-5_1

Gao Y, Hu X, Liu H, Feng Y (2010) Multiobjective estimation of distribution algorithm combined with pso for RFID network optimization. In: Measuring Technology and Mechatronics Automation (ICMTMA), 2010 International Conference on, vol 2, pp 736-739

Gong Y, Shen M, Zhang J, Kaynak O, Chen W, Zhan Z (2012) Optimizing RFID network planning by using a particle swarm optimization algorithm with redundant reader elimination. IEEE Trans Industrial Informatics 8(4):900-912

Guan Q, Liu Y, Yang Y, Yu W (2006) Genetic approach for network planning in the RFID systems. In: Intelligent Systems Design and Applications, 2006. ISDA '06. Sixth International Conference on, vol 2, pp 567-572

Hanning C, Yunlong Z, Kunyuan H, Tao K (2011) RFID network planning using a multi-swarm optimizer. Journal of Network and Computer Applications 34(3):888-901

Hasnan K, Talib NH, Nawawi A (2019) Analysis of gradient-based cuckoo search for the large scale optimal RFID network planning. Journal of Physics: Conference Series 1150:012008, doi:10.1088/1742-6596/1150/1/012008, URL https: //doi.org/10.1088\%2F $1742-6596 \% 2$ F $1150 \% 2$ F $1 \% 2$ F012008

hasnan KB, Talib NH, Nawawi AB, Elewe AM, Abdullah HB, Tahir S (2019) An efficient algorithm for large-scale rfid network planning. In: 2019 IEEE Jordan International Joint Conference on Electrical Engineering and Information 
Technology (JEEIT), pp 519-524

Jaballah A, Meddeb A (2017a) Algorithm for readers arrangement without collision in RFID networks. In: 2017 18th International Conference on Parallel and Distributed Computing, Applications and Technologies (PDCAT), pp 316-321

Jaballah A, Meddeb A (2017b) A new variant of cuckoo search algorithm with self adaptive parameters to solve complex RFID network planning problem. Wireless Networks

Jaballah A, Meddeb A (2017) Self adaptive cuckoo search algorithm for RFID network planning. In: 2017 Internet Technologies and Applications (ITA), pp 122-127, doi:10.1109/ITECHA.2017.8101922

Ma L, Hu K, Zhu Y, Chen H (2014) Cooperative artificial bee colony algorithm for multi-objective RFID network planning. Journal of Network and Computer Applications 42:143 - 162

Ma L, Wang X, Huang M, Lin Z, Tian L, Chen H (2019) Two-level master-slave RFID networks planning via hybrid multiobjective artificial bee colony optimizer. IEEE Transactions on Systems, Man, and Cybernetics: Systems 49(5):861-880, doi 10.1109/TSMC.2017.2723483

Meddeb A (2016) Internet of things standards: who stands out from the crowd? IEEE Communications Magazine 54(7):40-47

Nebojsa Bacanin MT, Strumberger I (2015) RFID network planning by ABC algorithm hybridized with heuristic for initial number and locations of readers. In: 17th UKSIM-AMSS International Conference on Modelling and Simulation, pp 39-44

Prud'homme C, Fages JG, Lorca X (2017) Choco Documentation. TASC - LS2N CNRS UMR 6241, COSLING S.A.S., URL http://www. choco-solver.org

Ruan W, Sheng QZ, Yao L, Li X, Falkner NJ, Yang L (2018) Device-free human localization and tracking with uhf passive rfid tags: A data-driven approach. Journal of Network and Computer Applications 104:78 - 96

Shi W, Guo Y, Yan S, Yu Y, Luo P, Li J (2018) Optimizing directional reader antennas deployment in UHF RFID localization system by using a MPCSO algorithm. IEEE Sensors Journal 18(12):5035-5048, doi: $10.1109 /$ JSEN.2018.2832216

Figueiredo e Silva P, Kaseva V, Lohan ES (2018) Wireless positioning in IoT: A look at current and future trends. Sensors 18(8)

Talib NH, Hasnan KB, Nawawi AB, Abdullah HB (2020) Monitoring largescale rail transit systems based on an analytic hierarchy process/gradientbased cuckoo search algorithm (gbcs) scheme. Urban Rail Transit 6:132-144, doi:https://doi.org/10.1007/s40864-020-00126-3

Tuba M, Bacanin N (2015) Hybridized bat algorithm for multi-objective radio frequency identification (rfid) network planning. In: 2015 IEEE Congress on Evolutionary Computation (CEC), pp 499-506

Tuba M, Bacanin N, Beko M (2015) Multiobjective rfid network planning by artificial bee colony algorithm with genetic operators. In: Advances in Swarm and Computational Intelligence, Springer International Publishing, pp 247-254

Xu B, Li J, Yang Y, Postolache O, Wu H (2018) Robust modeling and planning of radio-frequency identification network in logistics under uncertainties. International Journal of Distributed Sensor Networks 14(4):1550147718769781, doi: $10.1177 / 1550147718769781$ 
Yang Y, Wu Y, Xia M, Qin Z (2009) A RFID network planning method based on genetic algorithm. Networks Security, Wireless Communications and Trusted Computing, International Conference on 1:534-537

Yuan C, Hanning C, Shen J, Lin N, Su W, Liu F, Liang X (2018) Indicatorbased multi-objective adaptive bacterial foraging algorithm for RFID network planning. Cluster Computing doi:10.1007/s10586-018-1715-0, URL https : //doi.org/10.1007/s10586-018-1715-0

Zahran EG, Arafa AA, Saleh HI, Dessouky MI (2020) A self learned invasive weedmixed biogeography based optimization algorithm for rfid network planning. Wireless Networks doi:https://doi.org/10.1007/s11276-020-02316-0

Zhang T, Liu J (2017) An efficient and fast kinematics-based algorithm for RFID network planning. Computer Networks 121:13 - 24

Zhang W, Lin B, Gao C, Yan Q, Li S, Li W (2018) Optimal placement in RFIDintegrated VANETs for intelligent transportation system. In: 2018 IEEE International Conference on RFID Technology Application (RFID-TA), pp 1-6

Zhang Z, Zhang J, Wu L, Song H (2019) An improved approach for rfid network planning: Introduction of directional antenna reader. In: 2019 IEEE International Conference on Smart Manufacturing, Industrial Logistics Engineering (SMILE), pp 29-33

Zhao C, Wu C, Chai J, Wang X, Yang X, Lee JM, Kim MJ (2017) Decompositionbased multi-objective firefly algorithm for RFID network planning with uncertainty. Applied Soft Computing 55:549 - 564

Zhu W, Li M (2018) RFID reader planning for the surveillance of predictable mobile objects. Procedia Computer Science 129:475 - 481, doi:https://doi.org/10.1016/j.procs.2018.03.027, international conference on identification,information and knowledge in the internet of things

Zhu X, Mukhopadhyay SK, Kurata H (2012) A review of RFID technology and its managerial applications in different industries. Journal of Engineering and Technology Management 29(1):152 - 167 